\title{
A classification of solutions of a conformally invariant fourth order equation in $\mathbf{R}^{n}$
}

\author{
Chang-Shou Lin
}

Abstract. In this paper, we consider the following conformally invariant equations of fourth order

$$
\left\{\begin{array}{l}
\Delta^{2} u=6 e^{4 u} \quad \text { in } \mathbf{R}^{4}, \\
e^{4 u} \in L^{1}\left(\mathbf{R}^{4}\right)
\end{array}\right.
$$

and

$$
\left\{\begin{array}{l}
\Delta^{2} u=u^{\frac{n+4}{n-4}}, \\
u>0 \text { in } \mathbf{R}^{n} \text { for } n \geq 5,
\end{array}\right.
$$

where $\Delta^{2}$ denotes the biharmonic operator in $\mathbf{R}^{n}$. By employing the method of moving planes, we are able to prove that all positive solutions of (2) are arised from the smooth conformal metrics on $S^{n}$ by the stereograph projection. For equation (1), we prove a necessary and sufficient condition for solutions obtained from the smooth conformal metrics on $S^{4}$.

Mathematics Subject Classification (1991). 35J60.

Keywords. Elliptic equations of fourth order, conformal geometry.

\section{Introduction}

Recently, there have been much analytic work on the conformal geometry. A well known example is the Yamabe problem or, more generally, the problem of prescribling scalar curvature. Given a smooth function $K$ defined in a compact Riemannian manifold $\left(M, g_{0}\right)$ of dimension $n \geq 2$, we ask whether there exists a metric $g$ conformal to $g_{0}$ such that $K$ is the scalar curvature of the new metric $g$. Let $g=e^{2 u} g_{0}$ for $n=2$ or $g=u^{\frac{4}{n-2}} g_{0}$ for $n \geq 3$, then the problem is reduced to find solutions of the following nonlinear elliptic equations:

$$
\Delta u+K e^{2 u}=K_{0}
$$


for $n=2$, or,

$$
\left\{\begin{array}{l}
\frac{4(n-1)}{n-2} \Delta u+K u^{\frac{n+2}{n-2}}=K_{0} u, \\
u>0 \text { in } M
\end{array}\right.
$$

for $n \geq 3$, where $\Delta$ denotes the Beltrami-Laplacian operator of $\left(M, g_{0}\right)$ and $K_{0}$ is the scalar curvature of $g_{0}$. In studying equations (1.1) and (1.2), it is very important to understand the solution set of

$$
\left\{\begin{array}{l}
\Delta u+n(n-2) u^{\frac{n+2}{n-2}}=0 \text { in } \mathbf{R}^{n}, \\
u>0 \text { in } \mathbf{R}^{n}
\end{array}\right.
$$

for $n \geq 3$, or,

$$
\left\{\begin{array}{l}
\Delta u+e^{2 u}=0 \text { in } \mathbf{R}^{2} \\
e^{2 u} \in L^{1}\left(\mathbf{R}^{2}\right) .
\end{array}\right.
$$

By employing the method of moving planes, Caffarelli-Gidas-Spruck [CGS] was able to classify all the solutions of (1.3) for $n \geq 3$, and, Chen-Li [CL] did the same thing for the equation (1.4).

There are another interesting examples arising from the conformal geometry. For a compact Riemannian manifold of dimension 4, Chang and Yang [CY] considered the existence of extremal functions of the variational problem:

$$
I I[w]=<P w, w>+\int Q_{0} w d V_{g_{0}}-\left(\int Q_{0} d V_{g_{0}}\right) \log \int e^{4 w} d V_{g_{0}},
$$

where $P$ is the Paneitz operator on $M$, discovered by Paneitz:

$$
\begin{aligned}
& P \varphi=\Delta^{2} \varphi+\delta\left(\frac{2}{3} K_{0} I-2 R i c\right) d \varphi \\
& Q_{0}=\frac{1}{12}\left(K_{0}^{2}-\Delta K_{0}-3|R i c|^{2}\right)
\end{aligned}
$$

where Ric is the Ricci curvature of $\left(M, g_{0}\right)$. The variational form (1.5) arises form the difference of log-determinants of conformally covariant operator with respect to metrics in a conformal class. For background material and other related problems, we refer [BCY], [CY] and the references therein. The extremal function $u$ of $I I(w)$ satisfies a conformal invariant elliptic equation of fourth order:

$$
P u+2 Q_{0}=2 Q e^{4 u},
$$

where $Q$ is a constant. When $\left(M, g_{0}\right)$ is the standard $S^{4}$, by using the coordinate of the stereographic projection in $\mathbf{R}^{4}$, the equation (1.6) can be reduced to

$$
\left\{\begin{array}{l}
\Delta^{2} u=6 e^{4 u} \\
e^{4 u} \in L^{1}\left(\mathbf{R}^{4}\right)
\end{array} \text { in } \mathbf{R}^{4}\right.
$$


where $\Delta^{2}$ denotes the biharmonic operator. It is expected that in order to understand the equation (1.6), we should classify all the solutions of (1.7) completely.

The equation (1.7) looks very similar to the equation (1.4). In fact, there are many common properties shared by both equations. For example, the biharmonic operator $\Delta^{2}$ in $\mathbf{R}^{4}$ has const. $\log \frac{1}{|x-y|}$ as its fundamental solution. And the equation (1.7) is invariant under the change of the conformal transformation. In particular, the new function $w(x)=u\left(\frac{x}{|x|^{2}}\right)-2 \log |x|$ satisfies the same equation as $u$ does. However, the appearance of the biharmonic operator in (1.7) expects to make the equation (1.7) very different from (1.4). In fact, a study of radial solutions of (1.7) shows that there are solutions of (1.7) which do not come from the smooth functions on $S^{4}$ through the stereographic projection. This is not quite the same as the equation (1.4). But, under certain constraint on the behavior of $u$ at $\infty$, we have

Theorem 1.1. Suppose that $u$ is a solution of (1.7) with $|u(x)|=o\left(|x|^{2}\right)$ at $\infty$. Then there exists some point $x_{0} \in \mathbf{R}^{4}$ such that $u$ is radially symmetric about $x_{0}$ and

$$
u(x)=\log \frac{2 \lambda}{\left(1+\lambda^{2}\left|x-x_{0}\right|^{2}\right)} .
$$

Let $\alpha$ be defined by

$$
\alpha=\frac{3}{4 \pi^{2}} \int_{\mathbf{R}^{4}} e^{4 u(y)} d y .
$$

Theorem 1.2. Let $u$ be a solution of (1.7). Then the following statements hold. (i) After an orthorgonal transformation, $u(x)$ can be represented by

$$
\begin{aligned}
u(x) & =\frac{3}{4 \pi^{2}} \int_{\mathbf{R}^{4}} \log \left(\frac{|y|}{|x-y|}\right) e^{4 u(y)} d y-\sum_{j=1}^{4} a_{j}\left(x_{j}-x_{j}^{0}\right)^{2}+c_{0} \\
& =-\sum_{j=1}^{4} a_{j}\left(x_{j}-x_{j}^{0}\right)^{2}-\alpha \log |x|+c_{0}+O\left(|x|^{-\tau}\right)
\end{aligned}
$$

for some $\tau>0$ and for large $|x|$. The function $\Delta u$ satisfies

$$
\Delta u(x)=-\frac{3}{2 \pi^{2}} \int_{\mathbf{R}^{4}} \frac{e^{4 u(y)}}{|x-y|^{2}} d y-2 \sum_{j=1}^{4} a_{j}
$$

where $a_{j} \geq 0, c_{0}$ are constants and $x^{0}=\left(x_{1}^{0}, \ldots, x_{4}^{0}\right) \in \mathbf{R}^{4}$. Moreover, if $a_{i} \neq 0$ for all $i$, then $u$ is symmetric with respect to the hyperplane $\left\{x \mid x_{i}=x_{i}^{0}\right\}$. If $a_{1}=a_{2}=a_{3}=a_{4} \neq 0$, then $u$ is radially symmetric with respect to $x^{0}$.

(ii) The total integration $\alpha \leq 2$. If $\alpha=2$, then $u(x)$ has the form of (1.8). 
In this paper, we also consider the following equation analogue to the equation (1.3):

$$
\left\{\begin{array}{l}
\Delta^{2} u=u^{\frac{n+4}{n-4}} \\
u>0 \text { in } \mathbf{R}^{n}
\end{array}\right.
$$

for $n \geq 5$. The equation (1.12) can be derived from the Sobolev embedding of $H^{2}$ into $L^{\frac{2 n}{n-4}}$ :

$$
\sup _{u \in H^{2}\left(\mathbf{R}^{4}\right)} \frac{\int|\Delta u|^{2}}{\left(\int u^{\frac{2 n}{n-4}}\right)^{\frac{n-4}{n}}} .
$$

The existence of extremal functions of (1.13) was shown in [L] by P.L. Lions. In the same paper, Lions also proved the radial symmetry of any extremal function of (1.13). In general, the radial symmetry of solutions of (1.12) holds also.

Theorem 1.3. Suppose that $u$ is a smooth solution of (1.12). Then $u$ is radially symmetric about some point $x_{0} \in \mathbf{R}^{n}$ and $u$ has the following form:

$$
u(x)=c_{n}\left(\frac{\lambda}{1+\lambda^{2}\left|x-x_{0}\right|^{2}}\right)^{\frac{n-4}{2}}
$$

for some constant $\lambda>0$, where $c_{n}=[n(n-4)(n-2)(n+2)]^{-\frac{n-4}{8}}$.

Similarly, we also have

Theorem 1.4. Suppose that $u$ is a nonnegative solution of

$$
\Delta^{2} u=u^{p} \text { in } \mathbf{R}^{n}
$$

for $1<p<\frac{n+4}{n-2}$. Then $u \equiv 0$ in $\mathbf{R}^{n}$.

As in equations (1.3) and (1.4), we will use the method of moving planes to prove the radial symmetry. In our situation, however, the maximum principle can not directly be applied to $u$ without any information of $\Delta u$. Hence we have to get some informations about $\Delta u$ from equations (1.7) and (1.12). First, we are going to prove that for any solution of $(1.7), \Delta u(x)$ can be reprensented by

$$
\Delta u(x)=\frac{-3}{2 \pi^{2}} \int_{\mathbf{R}^{4}} \frac{e^{4 u(y)}}{|x-y|^{2}} d y-c_{1}
$$

for some nonnegative constant $c_{1} \geq 0$. Thus, $u$ satisfies $\Delta u<0$ in $\mathbf{R}^{4}$. The representation (1.16) is an indication that we should apply the method of moving planes to $-\Delta u$, not $u$ itself. The method of moving planes was first invented by A.D. Alexandrov, and was shown to be a powerful tool in studying equations (1.3) 
and (1.4) by Gidas-Ni-Nirenberg [GNN], Caffarelli-Gidas-Spruck [CGS], Chen-Li [CL] and many others. As usual, in order to start the process of moving planes at $\infty$, we have to understand the asymptotic behavior of both $u$ and $\Delta u$ at infinity. The analysis of asymptotic behaviors will be carried out in Section 2. In Section 3, we will establish the radial symmetry and prove Theorem 1.1 and Theorem 1.2. In Section 4, both Theorem 1.3 and Theorem 1.4 are proved.

The author would like to thank Professors Alice Chang and Paul Yang for introducing him to this problem. While preparing the manuscript, I was informed by Professor A. Chang that she and P. Yang have also obtained similar results by using the method of moving plane.

\section{Asymptotic behavior}

In this section, we want to study the asymptotic behavior for a solution $u$ of (1.7). First, we note that the fundamental solution of the biharmonic operator $\Delta^{2}$ in $R^{4}$ is

$$
P(x, y)=\frac{1}{8 \pi^{2}} \log \frac{1}{|x-y|} .
$$

Let $u$ be a solution of (1.7). Set

$$
\alpha=\frac{3}{4 \pi^{2}} \int_{\mathbf{R}^{4}} e^{4 u(y)} d y,
$$

and

$$
v(x)=\frac{3}{4 \pi^{2}} \int_{\mathbf{R}^{4}} \log \left(\frac{|x-y|}{|y|}\right) e^{4 u(y)} d y .
$$

Obviously, $v(x)$ satisfies

$$
\Delta^{2} v(x)=-e^{4 u(x)} \text { in } \quad R^{4}
$$

Lemma 2.1. Suppose $u$ is a solution of (1.7). Let $\alpha$ be given as in (2.1). Then there exists a constant $C$ such that

$$
v(x) \leq \alpha \log |x|+C
$$

Proof. For $|x| \geq 4$, we decompose $\mathbf{R}^{4}=A_{1} \cup A_{2}$, where $A_{1}=\left\{y|| y-x \mid \leq \frac{|x|}{2}\right\}$ and $A_{2}=\left\{y|| y-x \mid \geq \frac{|x|}{2}\right\}$. For $y \in A_{1}$, we have $|y| \geq|x|-|x-y| \geq \frac{|x|}{2} \geq|x-y|$, which implies

$$
\log \frac{|x-y|}{|y|} \leq 0
$$


Since $|x-y| \leq|x|+|y| \leq|x||y|$ for $|x|,|y| \geq 2$ and $\log |x-y| \leq \log |x|+C$ for $|x| \geq 4$ and $|y| \leq 2$, we have

$$
\begin{aligned}
v(x) & \leq \frac{3}{4 \pi^{2}} \int_{A_{2}} \log \frac{|x-y|}{|y|} e^{4 u(y)} d y \\
& \leq \frac{3}{4 \pi^{2}}\left(\log |x| \int_{A_{2}} e^{4 u(y)} d y+\int_{|y| \leq 2} \log \frac{|x-y|}{|y|} e^{4 u(y)} d y\right. \\
& \leq \frac{3}{4 \pi^{2}}\left(\int_{\mathbf{R}^{4}} e^{4 u(y)} d y\right) \log |x|+C \\
& =\alpha \log |x|+C .
\end{aligned}
$$

Lemma 2.2. Suppose $u$ is a solution of (1.7). Then $\Delta u(x)$ can be represented by

$$
\Delta u(x)=\frac{-3}{2 \pi^{2}} \int_{\mathbf{R}^{4}} \frac{e^{4 u(y)}}{|x-y|^{2}} d y-C_{1}
$$

where $C_{1} \geq 0$ is a constant.

Proof. Let $w(x)=u(x)+v(x)$. By (2.3), we have $\Delta^{2} w(x)=0$ in $\mathbf{R}^{4}$. Since $\Delta w(x)$ is a harmonic fumction in $\mathbf{R}^{4}$, we have for any $x_{0} \in \mathbf{R}^{4}$ and $r>0$,

$$
\begin{aligned}
\Delta w\left(x_{0}\right) & =\frac{2}{\pi^{2} r^{4}} \int_{\left|y-x_{0}\right| \leq r} \Delta w(y) d y \\
& =\frac{2}{\pi^{2} r^{4}} \int_{\left|y-x_{0}\right|=r} \frac{\partial w}{\partial r}(y) d \sigma .
\end{aligned}
$$

where $\pi^{2} / 2$ is the volume of the unit ball and $d \sigma$ denotes the area element of the sphere $\left|y-x_{0}\right|=r$.

Integrating (2.6) along $r$, we have

$$
\frac{r^{2}}{8} \Delta w\left(x_{0}\right)=f_{\left|x-x_{0}\right|=r} w d \sigma-w\left(x_{0}\right)
$$

where $f_{\left|x-x_{0}\right|=r} w d \sigma=\frac{1}{2 \pi^{2} r^{3}} \int_{\left|x-x_{0}\right|=r} w d \sigma$ is the integral average of $w$ over the sphere $\left|x-x_{0}\right|=r$. Hence, by the Jensen inequality,

$$
\begin{aligned}
\exp \left(\frac{r^{2}}{2} \Delta w\left(x_{0}\right)\right) & \leq e^{-4 w\left(x_{0}\right)} \exp \left(4 f_{\left|x-x_{0}\right|=r} w d \sigma\right) \\
& \leq e^{-4 w\left(x_{0}\right)} f_{\left|x-x_{0}\right|=r} e^{4 w} d \sigma .
\end{aligned}
$$


Since $w(x)=u(x)+v(x) \leq u(x)+\alpha \log |x|+c$, we have $r^{3-4 \alpha} \exp \left(\frac{\Delta w\left(x_{0}\right)}{2} r^{2}\right) \epsilon$ $L^{1}[1, \infty)$. Thus $\Delta w\left(x_{0}\right) \leq 0$ for all $x_{0} \in \mathbf{R}^{4}$. By Liouville's Theorem, $\Delta w(x) \equiv$ $-C_{1}$ in $\mathbf{R}^{4}$ for some constant $C_{1} \geq 0$. Hence (2.5) follows immediately.

Let $h(x)$ be the solution of

$$
\left\{\begin{array}{l}
\Delta^{2} h(x)=f(x) \text { in } \Omega \\
\Delta h(x)=h(x)=0 \text { on } \partial \Omega
\end{array}\right.
$$

where $\Omega$ is a bounded domain of $\mathbf{R}^{4}$. Following the argument of [BM], we have

Lemma 2.3. Suppose $f \in L^{1}(\bar{\Omega})$. For any $\delta \in\left(0,32 \pi^{2}\right)$, there exists a constant $C_{\delta}>0$ such that the inequality,

$$
\int_{\Omega} \exp \left(\frac{\delta|h|}{\|f\|_{L^{1}}}\right) d x \leq C_{\delta}(\operatorname{diam} \Omega)^{4},
$$

where diam $\Omega$ denotes the diameter of $\Omega$.

Proof. Without loss of generality, we may assume $0 \in \Omega$. Let $R=\operatorname{diam} \Omega$. Set

$$
v(x)=\frac{1}{8 \pi^{2}} \int_{B_{R}(0)} \log \left(\frac{2 R}{|x-y|}\right)|\tilde{f}(y)| d y
$$

where $\tilde{f}(y)=f(y)$ for $y \in \Omega$ and $\tilde{f}(y)=0$ for $y \notin \Omega$. By a direct computation, we have

$$
\Delta v(x)=\frac{-1}{4 \pi^{2}} \int_{B_{R}(0)}|x-y|^{-2}|\tilde{f}(y)| d y \leq 0
$$

for $x \in \Omega$. Since both $v$ and $-\Delta v$ are positive on $\partial \Omega$, we have by the maximum principle,

$$
|h(x)| \leq v(x) \text { for } x \in \Omega .
$$

Applying the Jensen inequality, we have

$$
\begin{aligned}
& \int_{\Omega} \exp \left(\frac{\delta|h(x)|}{\|f\|_{L^{1}}}\right) d x \\
& \quad \leq \int_{\Omega} \exp \left(\frac{\delta}{8 \pi^{2}} \int_{B_{R}(0)} \log \left(\frac{2 R}{|x-y|}\right) d \mu(y)\right) \\
& \quad \leq \int_{\Omega} \int_{B_{R}(0)}\left(\frac{2 R}{|x-y|}\right)^{\frac{\delta}{8 \pi^{2}}} d \mu(y) d x \leq C_{\delta} R^{4},
\end{aligned}
$$

where $d \mu(y)=\frac{1}{\|f\|_{L^{1}}}|\tilde{f}(y)| d y$. Hence Lemma 2.3 is proved. 
Lemma 2.4. Let $u$ be a solution of (1.7) and $v$ is defined by (2.2). Then, given any $\varepsilon>0$, there exists a $R=R(\varepsilon)$ such that for $|x| \geq R$, v(x) satisfies

$$
\begin{gathered}
v(x) \geq(\alpha-\varepsilon) \log |x|, \quad \text { and }, \\
\lim _{|x| \longrightarrow+\infty} \Delta v(x)=0 .
\end{gathered}
$$

Proof. To prove (2.9), we first claim that for any $\varepsilon>0$, there exists $R=R(\varepsilon)>0$ such that

$$
v(x) \geq\left(\alpha-\frac{\varepsilon}{2}\right) \log |x|+\frac{3}{4 \pi^{2}} \int_{B(x, 1)} \log |x-y| e^{4 u(y)} d y .
$$

To prove (2.11), we decompose $\mathbf{R}^{4}=A_{1} \cup A_{2} \cup A_{3}$, where $A_{1}=\left\{y|| y \mid<R_{0}\right\}$, $A_{2}=\left\{y|| x-y\left|\leq \frac{|x|}{2},\right| y \mid \geq R_{0}\right\}$, and $A_{3}=\left\{y|| x-y\left|\geq \frac{|x|}{2},\right| y \mid \geq R_{0}\right\}$. Let $R_{0}=R_{0}(\varepsilon)$ be sufficiently large such that

$$
\frac{3}{4 \pi^{2}} \int_{A_{1}} \log \frac{|x-y|}{|y|} e^{4 u(y)} d y \geq\left(\alpha-\frac{\varepsilon}{4}\right) \log |x|
$$

for large $|x|$.

For $|x|$ large, we have

$$
\begin{aligned}
\int_{A_{2}} & \log \left(\frac{|x-y|}{|y|}\right) e^{4 u(y)} d y \\
\quad & =\int_{A_{2}} \log |x-y| e^{4 u(y)} d y-\int_{A_{2}} \log |y| e^{4 u(y)} d y \\
& \geq \int_{B(x, 1)} \log |x-y| e^{4 u(y)} d y-\log (2|x|) \int_{A_{2}} e^{4 u(y)} d y .
\end{aligned}
$$

For $y \in A_{3}$ and $|y| \leq 2|x|$ we have $|x-y| \geq \frac{|x|}{2} \geq \frac{|y|}{4}$. For $x \in A_{3}$ and $|y| \geq 2|x|$ we have $|x-y| \geq|y|-|x| \geq \frac{|y|}{2}$. In any case, we have for $y \in A_{3}$,

$$
\frac{|x-y|}{|y|} \geq \frac{1}{4}
$$

Hence

$$
\int_{A_{3}} \log \frac{|x-y|}{|y|} e^{4 u(y)} d y \geq \log \left(\frac{1}{4}\right) \int_{A_{3}} e^{4 u(y)} d y .
$$

By (2.12), (2.13) and (2.14), we have (2.11) for large $|x|$. 
Let $0<\varepsilon_{0}<\pi^{2}$ and $R_{0}=R_{0}\left(\varepsilon_{0}\right)>0$ be sufficiently large such that

$$
6 \int_{B(x, 4)} e^{4 u} d y \leq \varepsilon_{0}
$$

for $|x| \geq R_{0}$. Let $h$ be the solution of

$$
\left\{\begin{array}{l}
\Delta^{2} h=6 e^{4 u(y)} \text { in } B(x, 4), \\
h=\Delta h=0 \text { on } \partial B(x, 4) .
\end{array}\right.
$$

By Lemma 2.3, we have for small $\varepsilon_{0}$,

$$
\int_{B(x, 4)} e^{12|h|} d y \leq c_{1}
$$

for some constant $c_{1}$ independent of $x$.

Set $q(y)=u(y)-h(y)$ for $y \in B(x, 4)$. Then $q$ satisfies

$$
\left\{\begin{array}{l}
\Delta^{2} q(y)=0 \text { on } B(x, 4), \\
\Delta q=\Delta u \text { and } q=u \text { on } \partial B(x, 4) .
\end{array}\right.
$$

Let $\tilde{q}(y)=-\Delta q(y)$. By Lemma 2.2, $\tilde{q}(y)$ is harmonic with positive boundary value on $\partial B(x, 2)$. Applying the maximum principle, we have $\tilde{q}(y)>0$ in $B(x, 4)$. Thus, by the Harnack inequality, we have

$$
\tilde{q}(y) \leq c_{2} \tilde{q}(x)=-c_{2} f_{\partial B(x, 4)} \Delta u d \sigma
$$

for $y \in \bar{B}(x, 2)$ where $c_{2}$ is a constant depending on $n$ only.

Integrating the equation (1.1), we have for any $r>0$,

$$
\int_{\partial B(x, r)} \frac{\partial}{\partial r}(\Delta u) d \sigma=6 \int_{B(x, r)} e^{4 u} d y .
$$

Integrating the identity above along $r$, we have

$$
f_{\partial B(x, r)} \Delta u-\Delta u(x)=\frac{3}{2 \pi^{2}} \int_{B(x, r)}\left(\frac{1}{|x-y|^{2}}-\frac{1}{r^{2}}\right) e^{4 u(y)} d y
$$

Applying Lemma 2.2 and (2.19), we have

$$
-f_{\partial B(x, r)} \Delta u=\frac{3}{2 \pi^{2}} \int_{|x-y| \geq r} \frac{e^{4 u(y)}}{|x-y|^{2}} d y+\frac{3}{2 \pi^{2} r^{2}} \int_{B(x, r)} e^{4 u(y)} d y+C_{1} .
$$


In particular, we have $r=4$,

$$
-f_{\partial B(x, 4)} \Delta u \leq c_{3}
$$

Hence, by (2.18), we have

$$
\tilde{q}(y) \leq c_{4} \text { for } y \in \overline{B(x, 2)} .
$$

Since $q$ satisfies

$$
\left\{\begin{array}{l}
\Delta q(y)=-\tilde{q}(y) \text { in } B(x, 4) \\
q=u \text { on } \partial B(x, 4)
\end{array}\right.
$$

by estimates for linear elliptic equations (e.g. see Theorem 8.17 in [GT]), we have for any $p>1$ and $\sigma>2$

$$
\sup _{B(x, 1)} q \leq c\left(\left\|q^{+}\right\|_{L^{p}(B(x, 2))}+\|\tilde{q}\|_{L^{\sigma}(B(x, 2))}\right),
$$

where $q^{+}=\max (q, 0)$ and $c=c(p, \sigma)$. Recall $q=u-h$. Thus, $q^{+}(y) \leq u^{+}(y)+$ $|h(y)|$ for $y \in B(x, 4)$. By (2.15), we have

$$
\int_{B(x, 2)} q^{+^{p}} \leq c_{5} \int_{B(x, 2)} e^{2 q^{+}} \leq c_{5}\left(\int_{B(x, 2)} e^{4 u^{+}}\right)^{\frac{1}{2}}\left(\int_{B(x, 2)} e^{4|h|}\right)^{\frac{1}{2}}
$$

Since $e^{4 u^{+}} \leq 1+e^{4 u}$, we have together with $(2.21)$,

$$
\sup _{B(x, 1)} q \leq c_{6} .
$$

Since $u=h+q$, we have

$$
u(y) \leq h(y)+q(y) \leq c_{6}+|h(y)|
$$

for $y \in B(x, 1)$. Therefore,

$$
\int_{B(x, 1)} e^{12 u} \leq c_{7} \int_{B(x, 1)} e^{12|h|} d y \leq c_{8}
$$

and then,

$$
\begin{aligned}
\left|\int_{B(x, 1)} \log \right| x-y\left|e^{4 u(y)} d y\right| \leq & \left(\int_{B(x, 1)}\left(\log \frac{1}{|x-y|}\right)^{2} d y\right)^{\frac{1}{2}} \\
& \left(\int_{B(x, 1)}\left(e^{8 u(y)} d y\right)^{\frac{1}{2}} \leq c_{9},\right.
\end{aligned}
$$


where $c_{9}$ is a constant in dependent of $x$. By (2.11), (2.9) follows immediately. By (2.24), it is an elementary exercise to prove $\lim _{|x| \longrightarrow \infty} \Delta v(x)=0$.

Lemma 2.5. Suppose $|u(x)|=o\left(|x|^{2}\right)$ at $\infty$. Then

$$
u(x)=\frac{3}{2 \pi^{2}} \int_{\mathbf{R}^{4}} \log \left(\frac{|y|}{|x-y|}\right) e^{4 u(y)} d y+C_{0}
$$

where $C_{0}$ is a constant. Furthermore, for any $\varepsilon>0$, there exists $R=R(\varepsilon)>0$ such that $u(x)$ satisfies

$$
-\alpha \log |x| \leq u(x) \leq(-\alpha+\varepsilon) \log |x|
$$

for $|x| \geq R(\varepsilon)$.

Proof. By Lemma 2.2, we have

$$
\Delta u(x)=\frac{-3}{2 \pi^{2}} \int_{2 \pi^{2}} \int_{\mathbf{R}^{4}}|x-y|^{-2} e^{4 u(y)} d y-C_{1} .
$$

Suppose $|u(x)|=o\left(|x|^{2}\right)$. First, we claim $C_{1}=0$. Otherwise, we have $\Delta u(x) \leq$ $-C_{1}<0$ for $|x| \geq R_{0}$ where $R_{0}$ is sufficiently large.

Let

$$
h(y)=u(y)+\varepsilon|y|^{2}+A\left(|y|^{-2}-R_{0}^{2-n}\right)
$$

where $\varepsilon$ is small such that

$$
\Delta h(y)=\Delta u+8 \varepsilon<-\frac{C_{1}}{2}<0
$$

for $|y| \geq R_{0}$, and $A$ is sufficiently large so that $\inf _{|y| \geq R_{0}} h(y)$ is achieved by some $y_{0} \in R^{4}$ with $\left|y_{0}\right|>R_{0}$. This can be done because $\lim _{|y| \longrightarrow+\infty} h(y)=+\infty$ for any $A>0$. Applying the maximum principle to $(2.28)$ at $y_{0}$, we have a contradiction. Hence the claim is proved.

By the claim, we have $\Delta(u+v)=0$ in $\mathbf{R}^{4}$. By the assumption and Lemma 2.1, we have $|u+v(x)|=o\left(|x|^{2}\right)$ at $\infty$. Since $u+v$ is a harmonic function, by the gradient estimates of harmonic functions, we have $u(x)+v(x)=\sum_{j=1}^{4} a_{j} x_{j}+a_{0}$ for some constants $a_{j} \in \mathbf{R}, 0 \leq j \leq 4$. Thus,

$$
e^{4 u(x)}=e^{a_{0}} e^{-4 v(x)} e^{\sum_{j=1}^{4} a_{j} x_{j}} \geq \text { const. }|x|^{-4 \alpha} e^{\sum_{j=1}^{4} a_{j} x_{j}} .
$$


Since $e^{4 u(x)} \in L^{1}\left(\mathbf{R}^{4}\right)$, we have $a_{j}=0$ for $1 \leq j \leq 4$. Hence, we have proved (2.25). Obviously, (2.26) immediately follows from (2.25), (2.9) and Lemma 2.1. The proof of Lemma 2.5 is finished.

Now suppose $u$ is a smooth solution of

$$
\Delta^{2} u=Q(x) e^{4 u} \text { in } \mathbf{R}^{4}
$$

where $Q(x) \in C^{1}\left(\mathbf{R}^{4}\right)$. Then we have the following Pohozaev identity.

Lemma 2.6. Suppose $u$ is an entire smooth function of (2.29). Then for any $R>0$, we have

$$
\begin{array}{rl}
\int_{B_{R}} & Q(x) e^{4 u} d x+\frac{1}{4} \int_{B_{R}}(x \cdot \nabla Q) e^{4 u} d x \\
& =\frac{1}{4} \int_{\partial B_{R}} Q(x)|x| e^{4 u} d \sigma-\int_{\partial B_{R}}|x|\left[\frac{(\Delta u)^{2}}{2}+|x| \frac{\partial u}{\partial r} \frac{\partial}{\partial r} \Delta u\right] d \sigma \\
& +\int_{\partial B_{R}} \frac{\partial}{\partial r}\left(r \frac{\partial u}{\partial r}\right) \Delta u d \sigma .
\end{array}
$$

Proof. The proof of Lemma 2.6 goes exactly the same as in the case of the semilinear elliptic equations of second order. For the sake of completeness, we give a proof here.

Multiplying $x \cdot \nabla u$ on the both sides of the equation (2.29), we have

$$
\begin{aligned}
\frac{1}{4} \int_{\partial B_{R}} Q|x| e^{4 u} d \sigma-\frac{1}{4}\left[\int_{B_{R}}[(x \cdot \nabla Q)+4 Q] e^{4 u} d x\right. \\
=\int_{B_{R}}(x \cdot \nabla u) Q e^{4 u} d x=\int_{B_{R}}(x \cdot \nabla u) \Delta^{2} u d x \\
=\int_{B_{R}} \Delta(x \cdot \nabla u) \Delta u d x+\int_{\partial B_{R}}\left[r \frac{\partial u}{\partial r} \frac{\partial}{\partial r}(\Delta u)-\Delta u \frac{\partial}{\partial r}\left(r \frac{\partial u}{\partial r}\right)\right] d \sigma \\
=\int_{\partial B_{R}}|x|\left[\frac{|\Delta u|^{2}}{2}+\frac{\partial u}{\partial r} \frac{\partial}{\partial r}(\Delta u)\right] d \sigma-\int_{\partial B_{R}} \Delta u \frac{\partial}{\partial r}\left(r \frac{\partial u}{\partial r}\right) d \sigma,
\end{aligned}
$$

where we have ultilized $\frac{1}{2} \operatorname{div}\left(x(\Delta u)^{2}\right)=\Delta(x \cdot \nabla u) \Delta u$. Obviously, (2.30) follows immediately.

Lemma 2.7. Let $u$ be a solution of (1.7) and $u(x)=o\left(|x|^{2}\right)$ at $\infty$. Then $\alpha=2$.

Proof. By Lemma 2.5, we have

$$
u(x)=\frac{3}{4 \pi^{2}} \int_{\mathbf{R}^{4}} \log \left(\frac{|y|}{|x-y|}\right) e^{4 u(y)} d y+C_{0} .
$$


By elementary calculations, we have

$$
\begin{aligned}
|x| \frac{\partial u}{\partial r}(x) & =-\frac{3}{4 \pi^{2}} \int_{\mathbf{R}^{4}} \frac{x \cdot(x-y)}{|x-y|^{2}} e^{4 u(y)} d y, \\
\frac{\partial}{\partial r}\left(r \frac{\partial u}{\partial r}\right)(x) & =-\frac{3}{4 \pi^{2}} \int_{\mathbf{R}^{4}} \frac{2 r^{2}-x \cdot y}{r|x-y|^{2}} e^{4 u(y)} d y \\
& +\frac{3}{2 \pi^{2}} \int_{\mathbf{R}^{4}} \frac{(x \cdot(x-y))^{2}}{r|x-y|^{4}} e^{4 u(y)} d y,
\end{aligned}
$$

and,

$$
\Delta u(x)=-\frac{3}{2 \pi^{2}} \int_{\mathbf{R}^{4}} \frac{e^{4 u(y)}}{|x-y|^{2}} d y
$$

Since $e^{4 u(y)} \geq|y|^{-4 \alpha}$ for large $|y|$ by lemma 2.5, we have $\alpha>1$. Therefore, it is easy to calculate from $(2.31) \sim(2.33)$ that

$$
\begin{gathered}
\lim _{|x| \longrightarrow+\infty}|x| \frac{\partial u}{\partial r}(x)=-\alpha, \\
\lim _{|x| \longrightarrow+\infty} \frac{\partial}{\partial r}\left(r \frac{\partial u}{\partial r}\right)(x)|x|=0, \\
\lim _{|x| \longrightarrow+\infty} \Delta u(x)|x|^{2}=-2 \alpha,
\end{gathered}
$$

and

$$
\lim _{|x| \longrightarrow+\infty} \frac{\partial}{\partial r}(\Delta u(x))|x|^{3}=4 \alpha .
$$

Applying the Pohozaev identity and $(2.34) \sim(2.37)$, the right hand side of (2.30) (Here, $Q(x)=6$ ) tends to $4 \pi^{2} \alpha^{2}$. Hence, we have

$$
8 \pi^{2} \alpha=4 \pi^{2} \alpha^{2}
$$

which implies $\alpha=2$.

Lemma 2.8. Let $u$ satisfy the assumption of Lemma 2.5. Then $u(x)$ satisfies

$$
u(x)=-2 \log |x|+c+O\left(|x|^{-1}\right)
$$

and

$$
\left\{\begin{array}{l}
-\Delta u(x)=4|x|^{-2}+\sum_{j=1}^{4} a_{j} x_{j}|x|^{-4}+O\left(x^{-4}\right) \\
-\frac{\partial}{\partial x_{i}} \Delta u(x)=-8 x_{i}|x|^{-4}+O\left(|x|^{-4}\right) \\
-\frac{\partial^{2}}{\partial x_{i} \partial x_{j}} \Delta u(x)=O\left(|x|^{-4}\right)
\end{array}\right.
$$


for large $|x|$, where $c, a_{j}, 1 \leq j \leq 4$ are constant.

Proof. Let $w(x)=u\left(\frac{x}{|x|^{2}}\right)-2 \log |x|$. By a straightforward computation, $w(x)$ satisfies

$$
\left\{\begin{array}{l}
\Delta^{2} w(x)=6 e^{4 w(x)} \text { in } \mathbf{R}^{4} \backslash\{0\} \\
|w(x)|=o\left(\log \frac{1}{|x|}\right) \text { and }|\Delta w(x)|=o\left(\frac{1}{|x|^{2}}\right) \text { as }|x| \longrightarrow 0 .
\end{array}\right.
$$

Set $h(x)$ be the solution of

$$
\left\{\begin{array}{l}
\Delta^{2} h(x)=6 e^{4 w(x)} \text { in } B_{1} \\
h(x)=w(x) \text { on } \partial B_{1}, \quad \Delta h(x)=\Delta w(x), \text { on } \partial B_{1} .
\end{array}\right.
$$

Since Lemma 2.5 implies $e^{4 w(x)} \in L^{p}\left(\bar{B}_{1}\right)$ for any $p>1$, by the regularity theorems of linear elliptic equations, $h(x) \in C^{3, \tau}\left(\bar{B}_{1}\right)$ for any $0<\tau<1$. Let $q(x)=w(x)-h(x)$. Then $q(x)$ satisfies

$$
\left\{\begin{array}{l}
\Delta^{2} q=0 \text { in } B_{1} \backslash\{0\} \\
q=\Delta q=0 \text { on } \partial B_{1} \\
|q(x)|=o\left(\log \frac{1}{|x|}\right),|\Delta q(x)|=o\left(\frac{1}{|x|^{2}}\right) \text { as }|x| \longrightarrow 0 .
\end{array}\right.
$$

By the maximum principle, we have, for any $\varepsilon>0$

$$
|\Delta q(x)| \leq \varepsilon /|x|^{2}
$$

for $x \in \bar{B}_{1}$. Applying the maximum principle again, we have

$$
|q(x)| \leq \varepsilon \log \frac{1}{|x|} .
$$

Thus, $q(x) \equiv 0$. Namely, $w(x)=h(x) \in C^{3, \tau}\left(\bar{B}_{1}\right)$. By the regularity of the linear elliptic equation again, we have $w(x) \in C^{\infty}\left(\bar{B}_{1}\right)$. It is not difficult to see that (2.39) follows immediately.

\section{Radial symmetry}

Now we are in the position to finish the proof of Theorem 1.1.

Proof of Theorem 1.1. Suppose that $u$ is a smooth entire solution of (1.7) such that $u(x)=o\left(|x|^{2}\right)$ at $\infty$. Let $v(x)=-\Delta u(x)$. By Lemma 2.8, $v(x)$ has a harmonic asymptotic expansion at $\infty$ :

$$
\begin{cases}v(x) & =\frac{1}{|x|^{2}}\left(4+\sum_{j=1}^{4} \frac{a_{j} x_{j}}{|x|^{2}}\right)+O\left(\frac{1}{|x|^{4}}\right) \\ v_{x_{i}} & =\frac{-8 x_{i}}{|x|^{4}}+O\left(\frac{1}{|x|^{4}}\right) \\ v_{x_{i} x_{j}} & =O\left(\frac{1}{|x|^{4}}\right)\end{cases}
$$


We want to apply the method of moving planes to prove that $u$ is symmetric about some point in $\mathbf{R}^{4}$. Following conventional notations, we let for any $\lambda, T_{\lambda}=\{x=$ $\left.\left(x_{1}, \ldots, x_{4}\right) \mid x_{1}=\lambda\right\}, \Sigma_{\lambda}=\left\{x \mid x_{1}>\lambda\right\}$ and $x^{\lambda}=\left(2 \lambda-x_{1}, x_{2}, \ldots, x_{4}\right)$ be the reflection point of $x$ with respect to $T_{\lambda}$. To start the process of moving planes along the $x_{1}$-direction, we need two lemmas below.

Lemma 3.1. Let $v$ be a positive function defined in a neighborhood at infinity satisfying the asymptotic expansion (3.1). Then there exists $\bar{\lambda}_{0}<0$ and $R>0$ such that the inequality

$$
v(x)>v\left(x^{\lambda}\right)
$$

holds for $\lambda \leq \bar{\lambda}_{0},|x| \geq R$ and $x \in \Sigma_{\lambda}$.

Lemma 3.2. Suppose $v$ satisfies the assumption of Lemma 3.1, and $v(x)>v\left(x^{\lambda_{0}}\right)$ for $x \in \Sigma_{\lambda_{0}}$. Assume $v(x)-v\left(x^{\lambda_{0}}\right)$ is superharmonic in $\Sigma_{\lambda_{0}}$. Then there exist $\varepsilon>0, S>0$ such that the followings hold.

(i) $v_{x_{1}}>0$ in $\left|x_{1}-\lambda_{0}\right|<\varepsilon$ and $|x|>S$.

(ii) $v(x)>v\left(x^{\lambda}\right)$ in $x_{1} \geq \lambda_{0}+\frac{\varepsilon}{2}>\lambda$ and $|x|>S$

for all $x \in \Sigma_{\lambda}, \lambda \leq \lambda_{1}$ with $\left|\lambda_{1}-\lambda_{0}\right|<c_{0} \varepsilon$, where $c_{0}$ is a small positive number depending on $\lambda_{0}$ and $v$ only.

The proofs of both lemmas are contained in [CGS]. Please see Lemma 2.3 and Lemma 2.4 in [CGS] for their proofs.

For any $\lambda$, we consider $w_{\lambda}(x)=u(x)-u\left(x^{\lambda}\right)$ in $\Sigma_{\lambda}$. Then $w_{\lambda}(x)$ satisfies

$$
\left\{\begin{array}{cl}
\Delta^{2} w_{\lambda}(x)=b_{\lambda}(x) w_{\lambda} & \text { in } \Sigma_{\lambda}, \\
w_{\lambda}(x)=\Delta w_{\lambda}(x)=0 & \text { on } T_{\lambda},
\end{array}\right.
$$

where $b_{\lambda}(x)=6 \frac{e^{4 u(x)}-e^{4 u\left(x^{\lambda}\right)}}{u(x)-u\left(x^{\lambda}\right)}>0$ in $\bar{\Sigma}_{\lambda}$. By Lemma 3.1, $\Delta w_{\lambda}(x)=v\left(x^{\lambda}\right)-$ $v(x)<0$ for $x \in \Sigma_{\lambda}, \lambda \leq \bar{\lambda}_{0}$ and $|x| \geq R$. Since $v(x)>0$ in $\mathbf{R}^{4}$, there exists $\bar{\lambda}_{1}<\bar{\lambda}_{0}$ such that

$$
v\left(x^{\lambda}\right)<v(x)
$$

for $|x| \leq R$ and $\lambda \leq \bar{\lambda}_{1}$. Therefore, we have

$$
\Delta w_{\lambda}(x)<0
$$

in $\Sigma_{\lambda}$ for $\lambda \leq \bar{\lambda}_{1}$. By Lemma 2.8, $\lim _{|x| \longrightarrow+\infty} w_{\lambda}(x)=0$. Applying the maximum principle, we have $w_{\lambda}(x)>0$ in $\Sigma_{\lambda}$ for all $\lambda \leq \overline{\lambda_{1}}$. 
Let $\lambda_{0}=\sup \left\{\lambda \mid v\left(x^{\mu}\right) \leq v(x)\right.$ for $x \in \Sigma_{\mu}$ and $\left.\mu \leq \lambda\right\}$. Since $v(x)$ tends to zero at $\infty$, it is not difficult to see that $\lambda_{0}<+\infty$. We claim that

$$
u(x) \equiv u\left(x^{\lambda_{0}}\right)
$$

for all $x \in \Sigma_{\lambda_{0}}$.

The claim will be proved by contradiction. Suppose $w_{\lambda_{0}} \not \equiv 0$ in $\Sigma_{\lambda_{0}}$. By continuity, $\Delta w_{\lambda_{0}}(x) \leq 0$ in $\Sigma_{\lambda_{0}}$. Since $w_{\lambda_{0}}(x)$ tends to 0 as $|x| \longrightarrow+\infty$ by (2.38), the strong maximum principle implies $w_{\lambda_{0}}(x)>0$ in $\Sigma_{\lambda_{0}}$. By applying equaiton (1.7), we have $\Delta^{2} w_{\lambda_{0}}(x)=6\left(e^{4 u(x)}-e^{4 u\left(x^{\lambda_{0}}\right)}\right)>0$, which implies $\Delta w_{\lambda_{0}}$ is a subharmonic function. Applying the strong maximum principle again, we have $\Delta w_{\lambda_{0}}(x)<0$ in $\Sigma_{\lambda_{0}}$.

By the definition of $\lambda_{0}$, there exists a sequence $\lambda_{n} \uparrow \lambda_{0}$ such that $\sup \Delta w_{\lambda_{n}}(x)>$ 0. Since $\lim _{|x| \longrightarrow+\infty} \Delta w_{\lambda_{n}}(x)=0$, there exists $x_{n} \in \Sigma_{\lambda_{n}}$ such that $\Delta w_{\lambda_{n}}\left(x_{n}\right)=$ $\sup \Delta w_{\lambda_{n}}(x)>0$. By Lemma 3.2. $x_{n}$ is bounded. Without loss of generality, $\Sigma_{\lambda_{n}}$ we may assume $x_{0}=\lim _{n \rightarrow+\infty} x_{n}$. If $x_{0} \in \Sigma_{\lambda_{0}}$, then by the continuity, we have $\Delta w_{\lambda_{0}}\left(x_{0}\right)=0$, which yields a contradition to $\Delta w_{\lambda_{0}}(x)<0$ in $\Sigma_{\lambda_{0}}$. If $x_{0} \in T_{\lambda_{0}}$, then $\nabla\left(\Delta w_{\lambda_{0}}\left(x_{0}\right)\right)=0$, which yields a contradiction to the Hopf boundary Lemma because $\Delta w_{\lambda_{0}}$ is a negative subharmonic function in $\Sigma_{\lambda_{0}}$. Therefore, the claim is proved. Obviously, the radial symmetry of $u$ follows from the claim.

By a straightforward computation, $u_{\lambda}(|x|) \equiv \log \left(\frac{2 \lambda}{1+\lambda^{2}|x|^{2}}\right)$ is a family of solutions of (1.7) for $\lambda>0$. Now let $\omega(r)$ be a radial solution of (1.7). From the uniqueness of ODE, $\omega(r)$ is completely determined by $\omega(0)$ and $\Delta \omega(0)=4 \omega^{\prime \prime}(0)$ ( $\omega$ always satisfies $\left.\omega^{\prime}(0)=\omega^{\prime \prime \prime}(0)=0\right)$. Without loss of generality, we may assume $\omega(0)=u_{\lambda_{0}}(0)$ for some $\lambda_{0}>0$. If $\omega^{\prime \prime}(0)<u_{\lambda_{0}}^{\prime \prime}(0)$, then $\omega(r)<u_{\lambda_{0}}(r)$ for small $r>0$. We first claim $u_{\lambda_{0}}(r)>\omega(r)$ for all $r>0$.

Suppose there exists $r_{0}>0$ such that $u_{\lambda_{0}}\left(r_{0}\right)=\omega\left(r_{0}\right)$ and $u_{\lambda_{0}}(r)>\omega(r)$ for $0 \leq r<r_{0}$. Then, by $(1.7)$,

$$
\frac{\partial}{\partial r} \Delta\left(u_{\lambda_{0}}(r)-\omega(r)\right)>0
$$

for $0<r \leq r_{0}$. In particular, $\Delta\left(u_{\lambda_{0}}(r)-\omega(r)\right)>0$ for $0 \leq r \leq r_{0}$. Since $u_{\lambda_{0}}(r)-\omega(r)=0$ on $r=r_{0}$, the maximum principle implies $u_{\lambda_{0}}(r)-\omega(r)<0$ for all $0 \leq r \leq r_{0}$, which yields a contradiction to $u_{\lambda_{0}}(0)=\omega(0)$. Thus, the claim is proved.

From the proof above, we also have $\Delta u_{\lambda_{0}}(r)-\Delta \omega(r)$ is increasing in $r$. Thus, $\omega(r) \sim-c r^{2}$ as $r \longrightarrow+\infty$ for some constant $c>0$.

If $\omega^{\prime \prime}(0)>u_{\lambda_{0}}^{\prime \prime}(0)$, then we have $\omega(r)>u_{\lambda_{0}}(r)$ for all $r>0$. By the equation (1.7), $\Delta \omega(r)-\Delta u_{\lambda_{0}}(r)$ is increasing in $r$. Thus, if $\omega(r)$ exists for all $r>0$ then 
$\omega(r) \geq c r^{2}$ for $r$ large and for some $c>0$. Hence, $\int_{\mathbf{R}^{4}} e^{4 \omega(|x|)} d x=+\infty$, and the proof of Theorem 1.1 is completely finished.

Lemma 3.3. Suppose that $u$ is a harmonic function in $\mathbf{R}^{n}$ such that $\exp (u-$ $\left.c|x|^{2}\right) \in L^{1}\left(\mathbf{R}^{n}\right)$ for some $c>0$. Then $u$ is a polynomial of order at most 2 .

Proof. For any unit vector $\xi \in \mathbf{R}^{4}$, we want to prove $u_{\xi \xi}(x) \equiv a$ constant. By Liouville's Theorem, it suffices to prove $u_{\xi \xi}(x)$ is bounded from above by a constant independent of $x$. Without loss of generality, we may take $x=0$ and $\xi=e_{1}$.

Since $u_{x_{1} x_{1}}$ is harmonic, we have for any $r>0$,

$$
\begin{aligned}
u_{x_{1} x_{1}}(0) & =\frac{1}{\sigma_{n} r^{n}} \int_{B_{r}(0)} u_{x_{1} x_{1}}(x) d x \\
& =\frac{1}{\sigma_{n} r^{n}} \int_{\partial B_{r}(0)} u_{x_{1}} \frac{x_{1}}{|x|} d \sigma
\end{aligned}
$$

where $\sigma_{n}$ is the volume of the unit ball in $\mathbf{R}^{n}$. Integrating the identity along $r$, we have

$$
\begin{aligned}
\frac{\sigma_{n}}{n+1} r^{n+1} u_{x_{1} x_{1}}(0) \\
\quad=\int_{B_{r}} u_{x_{1}} \frac{x_{1}}{|x|} d x \\
\quad=-\int_{B_{r}} u \frac{\partial}{\partial x_{1}}\left(\frac{x_{1}}{|x|}\right) d x+\int_{\partial B_{r}(0)} u \frac{x_{1}^{2}}{|x|^{2}} d \sigma \\
=-\int_{B_{r}} u\left(\frac{1}{|x|}-\frac{x_{1}^{2}}{|x|^{3}}\right) d x+\int_{\partial B_{r}(0)} u \frac{x_{1}^{2}}{|x|^{2}} d \sigma \\
=-\int_{B_{r}} \frac{u}{|x|} d x+\int_{B_{r}} u \frac{x_{1}^{2}}{|x|^{3}} d x+\int_{\partial B_{r}(0)} u \frac{x_{1}^{2}}{|x|^{2}} d \sigma
\end{aligned}
$$

The first integration can be written as

$$
\begin{aligned}
\int_{B_{r}} \frac{u}{|x|} d x & =\int_{0}^{s}\left(f_{\partial B_{s}(0)} u d \sigma\right)\left(n \sigma_{n}\right) s^{n-2} d s \\
& =n \sigma_{n} u(0) \int_{0}^{r} s^{n-2} d s \\
& =n \sigma_{n} u(0) \frac{r^{n-1}}{n-1} \\
& =\left(\frac{n \sigma_{n}}{n-1}\right) r^{n-1} u(0) .
\end{aligned}
$$


By a direct computation, we have

$$
\int_{B_{r}} \frac{x_{1}^{2}}{|x|^{3}} d x=\frac{\sigma_{n}}{n-1} r^{n-1}
$$

and

$$
\int_{\partial B_{r}} \frac{x_{1}^{2}}{|x|^{2}} d \sigma=\sigma_{n} r^{n-1}
$$

By (3.2), we have

$$
\frac{r^{2}}{n+1} u_{x_{1} x_{1}}(0)=-\frac{n}{n-1} u(0)+\frac{1}{n-1} f_{B_{r}(0)} u d \mu_{1}+f_{\partial B_{r}(0)} u d \mu_{2},
$$

where $d \mu_{1}=\frac{x_{1}^{2}}{|x|^{3}} d x$ and $d \mu_{2}=\nu_{1}^{2} d \sigma$ on $=\partial B_{r}(0)$. By Jensen's inequality, we have

$$
\begin{aligned}
& \exp \left(\frac{r^{2}}{2(n+1)} u_{x_{1} x_{1}}(0)\right) \\
& \quad \leq \exp \left(-\frac{n}{2(n-1)} u(0)\right)\left(f_{B_{r}(0)} e^{\frac{u}{2(n-1)}} d \mu_{1}\right) \cdot\left(f_{\partial B_{r}(0)} e^{\frac{u}{2}} d \mu_{2}\right)
\end{aligned}
$$

For any positive $c_{1}>0$, we have

$$
\begin{aligned}
\int_{1}^{\infty} & \exp \left[\left(\frac{1}{2(n+1)} u_{x_{1} x_{1}}(0)-c_{1}\right) r^{2}\right] d r \\
& \leq \exp \left(-\frac{n}{2 n-1} u(0)\right)\left(\int_{1}^{\infty}\left(f_{B_{r}(0)} u e^{\frac{u}{n-1}} d \mu_{1}\right) e^{-c_{1} r^{2}} d r\right)^{\frac{1}{2}} \\
& \left(\int_{1}^{\infty}\left(f_{\partial B_{r}(0)} u e^{u} d \mu_{1} e^{-c_{1} r^{2}} d r\right)^{\frac{1}{2}} .\right.
\end{aligned}
$$

By the assumption, we can choose a large $c_{1}$ such that the right hand side of (3.6) is finite. Thus, we have

$$
u_{x_{1} x_{1}}(0) \leq 2(n+1) c_{1} .
$$

By Liouville's Theorem, we have $u_{x_{1} x_{1}}(x) \equiv$ constant. Obviously, the conclusion of Lemma 3.3 follows immediately.

Proof of Theorem 1.2. Suppose that $u$ is a solution of (1.7) with $e^{4 u} \in L^{1}\left(\mathbf{R}^{4}\right)$. Let

$$
v(x)=\frac{3}{4 \pi^{2}} \int_{\mathbf{R}^{4}} \log \left(\frac{|x-y|}{|y|}\right) e^{4 u(y)} d y,
$$


and $w(x) \equiv u(x)+v(x)$. By Lemma 2.2, we have $\Delta w(x) \equiv-C_{1}$ in $\mathbf{R}^{4}$. Applying Lemma 3.3, we have $w(x) \equiv \Sigma\left(a_{i j} x_{i} x_{j}+b_{k} x_{k}\right)+c_{0}$, where $a_{i j}=a_{j i}$. After a change of coordinate by an orthorgonal transformation, we may assume

$$
u(x)=\frac{3}{4 \pi^{2}} \int_{\mathbf{R}^{4}} \log \frac{|y|}{|x-y|} e^{4 u(y)} d y-\sum_{i=1}^{4}\left(a_{i} x_{i}^{2}+b_{i} x_{i}\right)+c_{0},
$$

where $a_{i} \geq 0, b_{i}$ and $c_{0}$ are constants. Since $e^{4 u} \in L^{1}\left(\mathbf{R}^{4}\right)$, we have $b_{i}=0$ whenever $a_{i}=0$. Thus $u(x)$ can be written as

$$
u(x)=\frac{3}{4 \pi^{2}} \int_{\mathbf{R}^{4}} \log \frac{|y|}{|x-y|} e^{4 u(y)} d y-\sum_{i=1}^{n} a_{i}\left(x_{i}-x_{i}^{0}\right)^{2}+c_{0}
$$

After a translation, we may assume $x^{0}=0$. Let $\tilde{u}(x) \equiv u(x)+\sum_{i=1}^{n} a_{i} x_{i}^{2}$. Then $\tilde{u}(x)$ satisfies

$$
\Delta^{2} \tilde{u}(x)=Q(x) e^{4 \tilde{u}(x)} \text { in } \mathbf{R}^{4}
$$

where $Q(x)=6 e^{-4 \sum_{i=1}^{n} a_{i} x_{i}^{2}}$.

If $a_{i}=0$ for all $i$, then it is the case of Theorem 1.1. Thus, we assume $a_{i} \neq 0$ for $1 \leq i \leq k, a_{i}=0$ for $i>k$ where $1 \leq k \leq 4$. Lemma 2.1 implies $\alpha>1-\frac{k}{4}$. As in Lemma 2.8, we let $\tilde{w}(x)=u\left(\frac{x}{|x|^{2}}\right)-\alpha \log |x|=o\left(\log \frac{1}{|x|}\right)$. Then $\tilde{w}$ satisfies

$$
\Delta \tilde{w}+\tilde{Q}(x) e^{4 \tilde{w}}=0 \text { in } \mathbf{R}^{4} \backslash\{0\}
$$

where $\tilde{Q}(x)=6 e^{-\sum_{j} a_{j}\left(\frac{x_{j}}{|x|^{2}}\right)^{2}}|x|^{4(\alpha-2)}$.

Since $\alpha>1-\frac{k}{4}$, we have $\tilde{Q}(x) e^{4 \tilde{w}} \in L^{p}\left(B_{1}\right)$ for some $p>1$. By the same proof of Lemma 2.8 , we have $\tilde{w} \in C^{0, \tau}\left(\bar{B}_{1}\right)$ for some $1>\tau>0$. In particular, we have

$$
\tilde{u}(x)=-\alpha \log |x|+c_{0}+o\left(|x|^{-r}\right)
$$

at $\infty$, which together with (3.7), yields (1.10).

If $a_{i}<0$ for all $i$, then it is easily to see $\tilde{Q}(x) e^{4 \tilde{w}} \in L^{p}\left(\bar{B}_{1}\right)$ for any $p>1$. Thus $\tilde{w} \in C^{\infty}\left(\bar{B}_{1}\right)$. Therefore, $\tilde{u}$ satisfies both (2.38) and (2.39) for large $|x|$, i.e., we have for large $|x|$,

$$
\begin{gathered}
\tilde{u}(x)=-\alpha \log |x|+c_{0}+O\left(|x|^{-1}\right) \\
\left\{\begin{array}{l}
-\Delta \tilde{u}(x)=\frac{2 \alpha}{|x|^{2}}+\sum_{j=1}^{n} \frac{c_{j} x_{j}}{|x|^{4}}+O\left(|x|^{-4}\right) \\
-(\Delta \tilde{u})_{x_{i}}(x)=-\frac{4 \alpha x_{i}}{|x|^{4}}+O\left(|x|^{-4}\right) \\
(\Delta \tilde{u})_{x_{i} x_{j}}=O\left(|x|^{-4}\right)
\end{array}\right.
\end{gathered}
$$


Employing (3.10) and (3.11), we can use, as in the proof of Theorem 1.1, the method of moving planes to show that $\tilde{u}(x)$ is symmetric with respect to hyperplane $\left\{x \mid x_{i}=0\right\}$ for $1 \leq i \leq 4$. In particular, if $a_{1}=\ldots=a_{4} \neq 0$, then $u$ is radially symmetric with respect to 0 . Hence, we have proved (i) of Theorem 1.2.

If $\alpha \geq 2$, then $\tilde{Q}(x) e^{4 \tilde{w}} \in L^{p}\left(\bar{B}_{1}\right)$ for any $p>1$ also. Therefore $\tilde{w} \in C^{\infty}\left(\bar{B}_{i}\right)$, and, $e^{4 \tilde{u}}=O\left(|x|^{-8}\right)$ at $\infty$. By (2.31) $\sim(2.33)$, we can prove without difficulty:

$$
\begin{gathered}
\lim _{|x| \longrightarrow+\infty}|x| \frac{\partial \tilde{u}}{\partial r}(x)=-\alpha, \\
\lim _{|x| \longrightarrow+\infty} r \frac{\partial}{\partial r}\left(r \frac{\partial \tilde{u}}{\partial r}\right)(x)=0 \\
\lim _{r \longrightarrow+\infty} \Delta \tilde{u}(x)|x|^{2}=-2 \alpha
\end{gathered}
$$

and,

$$
\lim _{r \longrightarrow+\infty} \frac{\partial}{\partial r}(\Delta \tilde{u}) r^{3}=4 \alpha
$$

Applying the Pohozaev identity, we have

$$
8 \pi^{2} \alpha+\frac{1}{32 \pi^{2}} \int_{\mathbf{R}^{4}}(x, \nabla Q) e^{4 \tilde{u}} d x=4 \pi^{2} \alpha^{2}
$$

Since $\alpha \geq 2$, we have $8 \pi^{2} \alpha \leq 4 \pi^{2} \alpha^{2}$. Thus,

$$
\int_{\mathbf{R}^{4}}(x, \nabla Q) e^{4 u} d x \geq 0 .
$$

Since

$$
x \cdot \nabla Q=-\sum a_{j} x_{j}^{2} e^{-\Sigma a_{j} x_{j}^{2}} \leq 0,
$$

we have $a_{j}=0$ for all $j$. Then, by Theorem 1.1, we have $\alpha=2$ and $u(x)$ has a form of (1.8). Hence, (ii) of Theorem 1.2 is proved.

\section{Proof of Theorem 1.3}

Let $u$ be a smooth positive solution of

$$
\Delta^{2} u=u^{p} \text { in } \mathbf{R}^{n},
$$

for $1<p \leq \frac{n+4}{n-4}$ and $n \geq 5$. As in the case of the equation (1.3), we let

$$
u^{*}(x)=|x|^{4-n} u\left(\frac{x}{|x|^{2}}\right)
$$


By a direct computation, $u^{*}$ satisfies

$$
\Delta^{2} u^{*}=|x|^{-\tau} u^{* p} \text { in } \mathbf{R}^{n} \backslash\{0\},
$$

where $\tau=n+4-p(n-4) \geq 0$. Let $v(x)=-\Delta u^{*}(x)$. By (4.1), we have

$$
\left\{\begin{array}{l}
v(x)=c_{0}|x|^{2-n}+\sum_{j=1}^{n} \frac{a_{j} x_{j}}{|x|^{n}}+O\left(\frac{1}{|x|^{n}}\right) \\
v_{x_{i}}=-(n-2) c_{0}|x|^{-n} x_{i}+O\left(\frac{1}{|x|^{n}}\right) \\
v_{x_{i} x_{j}}=O\left(\frac{1}{|x|^{n}}\right)
\end{array}\right.
$$

at $\infty$, where $c_{0}>0$ and $a_{j} \in \mathbf{R}$. In particular, we have for large $|x|$,

$$
\Delta u^{*}(x)<0 .
$$

As in Theorem 1.1, we need to prove $\Delta u^{*}(x)<0$ in $\mathbf{R}^{n} \backslash\{0\}$.

Lemma 4.1. Let $u$ be a smooth positive solution of

$$
\Delta^{2} u=|x|^{-\tau} u^{p} \quad \text { in } B_{1} \backslash\{0\}
$$

where $1<p \leq \frac{n+4}{n-4}, \tau=(n+4)-p(n-4)$ and $n \geq 5$. Then $\Delta u$ is a subharmonic function in $B_{1}$ in the distributional sense.

Proof. First, we want to prove $|x|^{-\tau} u^{p} \in L^{1}\left(\bar{B}_{\frac{1}{2}}\right)$. Suppose $|x|^{-\tau} u^{p} \notin L^{1}\left(\bar{B}_{\frac{1}{2}}\right)$. Then we have

$$
\int_{\partial B_{r}} \frac{\partial}{\partial r}(\Delta u) d \sigma-\int_{\partial B_{s}} \frac{\partial}{\partial r}(\Delta u) d \sigma=\int_{B_{r} \backslash B_{s}}|x|^{-\tau} u^{p}>0
$$

for all $0<s \leq r \leq \frac{1}{2}$. Since the right hand side of (4.6) tends to $+\infty$ as $s \longrightarrow 0$, there exists $r_{1}>0$ such that

$$
f_{\partial B_{r}} \frac{\partial}{\partial r}(\Delta u) d \sigma \leq-c_{1} r^{1-n} \int_{B_{\frac{1}{2}} \backslash B_{r}}|x|^{-\tau} u^{p},
$$

which implies

$$
\int_{\partial B_{r}} \Delta u d \sigma-\int_{\partial B_{s}} \Delta u d \sigma \leq-c_{1} \int_{s}^{r} \tau^{1-n} \int_{B_{\frac{1}{2}} \backslash B_{r}}|x|^{-\tau} u^{p} d x d \tau,
$$

and

$$
\int_{\partial B_{r}} \Delta u d \sigma \geq c_{2} r^{-n+2}
$$


for $0<r \leq r_{2}$ and for some $0<r_{2}<r_{1}$. Let $\bar{u}=f_{\partial B_{r}} u d \sigma$. By (4.9),

$$
\left(r^{n-1} \bar{u}^{\prime}(r)\right)^{\prime} \geq c_{2} r
$$

If $\lim _{r \longrightarrow 0} r^{n-1} \bar{u}^{\prime}(r) \geq 0$, then we have for any $r>0$,

$$
r^{n-1} \bar{u}^{\prime}(r) \geq \frac{1}{2} c_{2} r^{2}
$$

which yields,

$$
\bar{u}(r) \geq \int_{0}^{r} \bar{u}^{\prime}(t) d t \geq \frac{1}{2} c_{2} \int_{0}^{r} t^{3-n} d t=+\infty,
$$

a contradiction. Therefore we may assume there exists $0<r_{3}<r_{2}$ such that for all $r \leq r_{3}$, we have

$$
r^{n-1} \bar{u}^{\prime}(r) \leq-c_{3},
$$

where $c_{3}$ is a positive constant. Therefore,

$$
\bar{u}(r) \geq c_{4} r^{2-n}
$$

Suppose $\bar{u}(r) \geq c_{4} r^{-s}$ for some $\sigma \geq n-2$. Then, by (4.7) and (4.8), we have for small $r>0$,

$$
\begin{gathered}
(\Delta \bar{u}(r))^{\prime} \leq-c_{1} r^{-\tilde{\sigma}-n+2}, \\
\Delta \bar{u}(r) \geq c_{2} r^{-\tilde{\sigma}-n+3}, \\
r^{n-1} \bar{u}^{\prime}(r) \leq-c_{3} r^{-\tilde{\sigma}+3},
\end{gathered}
$$

and,

$$
\bar{u}(r) \geq c_{4} r^{-\tilde{\sigma}+5-n},
$$

where $\tilde{\sigma}=\tau+p \sigma$. We note that, in order to have (4.16) held, we need $\tilde{\sigma}>3$. Since $\tau=n+4-p(n-4)$ and $\sigma \geq n-2$, we have $\tilde{\sigma} \geq \tau+p(n-2) \geq n+6>5$. Since $\tilde{\sigma}+n-5-p \sigma=\tau+n-5 \geq 0$ for $n \geq 5$, after a finite time of iterations, we have

$$
\begin{gathered}
\bar{u}(r) \geq r^{-(1+\beta),} \\
\bar{u}^{\prime}(r) \leq-r^{-(2+\beta)}, \\
\bar{u}^{\prime \prime}(r) \geq r^{-(3+\beta)},
\end{gathered}
$$

and,

$$
\bar{u}^{\prime \prime \prime}(r) \leq-r^{-(4+\beta)}
$$


for $0 \leq r \leq 2 r_{0}$ where $\beta=\frac{4}{p-1}$ (Since $\bar{u}^{\prime} \leq 0$, we have $0<\Delta \bar{u}(r) \leq \bar{u}^{\prime \prime}$ and $\left.\bar{u}^{(3)}(r) \leq(\Delta \bar{u})^{\prime}<0\right)$. By $(4.19) \sim(4.21)$, we have by Jensen inequality,

$$
\bar{u}^{(4)}(r) \geq \Delta^{2} \bar{u}(r) \geq|x|^{-\tau} \bar{u}^{p}(r) .
$$

Let $v(r)=A\left(r-r_{0}\right)^{-\beta}$ for $r_{0} \leq r \leq 2 r_{0}$. By direct computations, we have

$$
\begin{aligned}
v^{(4)}(r) & =A \beta(\beta+1)(\beta+2)(\beta+3)\left(r-r_{0}\right)-(\beta+4) \\
& =A \beta(\beta+1)(\beta+2)(\beta+3) v^{p}(r)<r^{-\tau} v^{p}(r)
\end{aligned}
$$

for $r_{0} \leq r \leq 2 r_{0}$ if $A$ is large. If $r_{0}$ is sufficiently small, then by (4.18) $\sim(4.21)$, we have $v(r) \leq \bar{u}(r)$ for all $r_{0} \leq r \leq 2 r_{0}$. However, $\lim _{r \longrightarrow r_{0}} \bar{u} \geq \lim _{r \longrightarrow r_{0}} v(r)=+\infty$ yields a contradiction. Therefore, we have proved $|x|^{-\tau} u^{p} \in L^{1}\left(\bar{B}_{\frac{1}{2}}\right)$.

Let $\varphi \in C_{0}^{\infty}\left(B_{\frac{1}{2}}\right)$ be a nonnegative function. We want to prove

$$
\int \Delta \varphi \Delta u d x \geq 0
$$

Let $\eta_{\varepsilon} \in C_{0}^{\infty}\left(B_{\frac{1}{2}}\right)$ satisfy $\eta_{\varepsilon}(x) \equiv 1$ for $|x| \geq 2 \varepsilon$, and $\eta_{\varepsilon}(x) \equiv 0$ for $|x| \leq \varepsilon$. We also assume

$$
\left|D^{j} \eta_{\varepsilon}(x)\right| \leq \frac{c}{\varepsilon^{j}}
$$

for $1 \leq j \leq 4$. Multiplying (4.5) by $\varphi(x) \eta_{\varepsilon}$, we have

$$
\begin{aligned}
0 & <\int \varphi(x) \eta_{\varepsilon}(x)|x|^{-\tau} u^{p}(x) d x \\
& =\int \Delta\left(\varphi(x) \eta_{\varepsilon}(x)\right) \Delta u(x) d x \\
& =\int \Delta u(x)\left\{\Delta \varphi(x) \eta_{\varepsilon}(x)+2 \nabla \varphi(x) \nabla \eta_{\varepsilon}+\varphi(x) \Delta \eta_{s}\right\} d x
\end{aligned}
$$

Let $\psi(x)=2 \nabla \varphi(x) \nabla \eta_{\varepsilon}+\varphi(x) \Delta \eta_{\varepsilon}(x)$. We have $\psi(x) \equiv 0$ for $|x| \leq \varepsilon$ and for $|x| \geq 2 \varepsilon$, and $|\Delta \psi(x)| \leq c \varepsilon^{-4}$.

Since $\frac{n}{q}+\frac{\tau}{p}=\frac{4}{p}+4>4$ where $\frac{1}{q}=1-\frac{1}{p}$, we have

$$
\begin{aligned}
\left|\int \Delta u(x) \psi(x) d x\right| & \leq \int u(x)|\Delta \psi(x)| d x \\
& \leq c \varepsilon^{-4}\left(\int_{\varepsilon \leq|x| \leq 2 \varepsilon}|x|^{-\tau} u^{p}(x) d x\right)^{1 / p} \varepsilon^{\frac{n}{q}+\frac{\tau}{p}} \\
& \leq c \varepsilon^{\frac{n}{q}+\frac{\tau}{p}-4} \longrightarrow 0,
\end{aligned}
$$


as $\varepsilon \longrightarrow 0$. Therefore, by (4.24), we have

$$
\begin{aligned}
\int \Delta u(x) \Delta \varphi(x) d x & =\lim _{\varepsilon \underset{\longrightarrow}{\longrightarrow} \int} \int \eta_{\varepsilon}(x) \Delta u(x) \Delta \varphi(x) d x \\
& =\int \varphi(x)|x|^{-\tau} u^{p}(x) d x>0
\end{aligned}
$$

Thus, $\Delta u(x)$ is a subharmonic in $B_{\frac{1}{2}}$.

Proofs of Theorem 1.3. and Theorem 1.4. Let $v(x)=-\Delta u^{*}(x)$. By Lemma 4.1 and (4.3), we have $v(x)>0$ in $\mathbf{R}^{4} \backslash\{0\}$ and $v(x)$ satisfies for any $r>0$,

$$
v(x) \geq \inf _{\partial B_{r}(0)} v(x)>0, \text { for } x \in B_{r}(0) .
$$

Since $u^{*}(x)$ is a superharmonic function in $B_{r}(0) \backslash\{0\}$ and $u^{*}(x)>0$, then we have

$$
u^{*}(x) \geq \inf _{\partial B_{r}(0)} u^{*}(y) \text { for } x \in B_{r}(0)
$$

(For a proof of (4.26), please see Lemma 2.1 in [CLn]).

Following notations in Section 3, we let $w_{\lambda}(x)=u^{*}(x)-u^{*}\left(x^{\lambda}\right)$ in $\Sigma_{\lambda}$. Since $v(x)=-\Delta u^{*}$ has a harmonic expansion (4.3) at infinity, by Lemma 3.1 and (4.25), there exists a $\bar{\lambda}_{0}<0$ such that

$$
\Delta w_{\lambda}(x)<0 \text { in } \Sigma_{\lambda}
$$

for all $\lambda \leq \overline{\lambda_{0}}$. By the maximum principle, we have

$$
w_{\lambda}(x)>0 \text { in } \Sigma_{\lambda}
$$

for all $\lambda \leq \bar{\lambda}_{0}$

We consider the case $p<\frac{n+4}{n-4}$ first. Let

$$
\lambda_{0}=\sup \left\{\lambda<0 \mid \Delta w_{\mu}(x)<0 \text { in } \Sigma_{\mu} \text { for } \mu \leq \lambda\right\} .
$$

Suppose $\lambda_{0}<0$. Although $u^{*}$ may has a singularity at 0 , by (4.25) and (4.26), we still can apply the same arguments as in Theorem 1.1 to prove $w_{\lambda_{0}}(x) \equiv 0$ in $\Sigma_{\lambda_{0}}$. Since $\tau<0$, it yields a contradiction. Thus we must have $\lambda_{0}=0$ and

$$
u\left(-x_{1}, x_{2}, \ldots, x_{n}\right) \leq u\left(x_{1}, x_{2}, \ldots, x_{n}\right) \text { for } x_{1} \geq 0
$$

By applying the method of moving planes along any direction in $\mathbf{R}^{n}, u^{*}(x)$ is radially symmetric with respect to 0 . Since we can take any point in $\mathbf{R}^{n}$ as the 
origin, we conclude that if $u$ is a positive smooth solution in $\mathbf{R}^{n}$, then $u \equiv$ constant in $\mathbf{R}^{n}$ which implies $u \equiv 0$ in $\mathbf{R}^{n}$, a contradiction. Thus, Theorem 1.4 is proved.

For the case $p=\frac{n+4}{n-4}$, we also let

$$
\lambda_{0}=\sup \left\{\lambda<0 \mid \Delta w_{\mu}(x)<0 \text { in } \Sigma_{\mu} \text { for } \mu \leq \lambda\right\} .
$$

If $\lambda_{0}<0$, by applying the same arguments again, we can show $w_{\lambda_{0}}(x) \equiv 0$. Thus, $u^{*}(x)$ has a removable singularity at 0 and $u$ itself satisfies (4.3) at infinity. Therefore, we can directly apply the method of moving plane to $u$ itself to yield the radial symmetry of $u$ about some point $x_{0}$ in $\mathbf{R}^{n}$. If $\lambda_{0}=0$, then we can do the same procedure by moving the hyperplane $T_{\lambda}$ from positive direction of $x_{1}$. Thus, we can prove either $u^{*}$ has a removable singularity at 0 or $u^{*}(x)$ is symmetric with respect to the hyperplane $\left\{x \mid x_{1}=0\right\}$. In any case, the radial symmetry of $u$ follows immediately.

Suppose that $u$ is radially symmetric with respect to 0 . We can take another point $x_{0} \neq 0$ as the origin of the "Kelvin" transformation, and do the same procedure as the above. Since $u$ is not radially symmetric about $x_{1}$, we have $\lambda_{0} \neq 0$, namely, $u(x)$ satisfies $(4.3)$ at infinity. In particular, we have $\Delta u(x) \longrightarrow 0$ as $|x| \longrightarrow+\infty$.

By a direct computation, we can see that $u_{\lambda}(x)=c_{n}\left(\frac{\lambda}{1+\lambda^{2}|x|^{2}}\right)^{\frac{n-4}{2}}$ is a solution of (1.12) for any $\lambda>0$. Suppose $\omega(r)$ is a radial solution of (1.12) and $\omega(0)=u_{\lambda_{0}}(0)$ for some $\lambda_{0}>0$. If $\Delta \omega(0)>\Delta u_{\lambda_{0}}(0)$, then we can prove $\omega(r)$ blows up in finite $r$. Because, if $\omega(r)$ exists for all $r>0$, as in the proof of Theorem 1.1, then we can show $\omega(r)>u_{\lambda_{0}}(r)$ for all $r>0$ and $(\Delta \omega-\Delta u)^{\prime}(r)>0$ for all $r>0$. Therefore

$$
\Delta \omega(\infty)=\lim _{r \longrightarrow+\infty}\left(\Delta \omega-\Delta u_{\lambda_{0}}\right)(r)>\left(\Delta \omega-\Delta u_{\lambda_{0}}\right)(0)>0
$$

which yields a contradition to $\lim _{|x| \longrightarrow+\infty} \Delta \omega(x)=0$ which was already proved for any solution of (1.12). If $\Delta \omega(0)<\Delta u_{\lambda_{0}}(0)$, then, by the same proof, $\omega(r)$ must become zero at a finite $r$. Thus the proof of Theorem 1.3 is considered completely finished.

In fact, the same proof can imply

Theorem 4.2. Suppose $u$ is a positive smooth solution of

$$
\Delta^{2} u=u^{p} \text { in } R^{n} \backslash\{0\}
$$

where $1<p \leq \frac{n+2}{n-4}$. Assume 0 is a nonremovable singularity, then $u$ is radially symmetric with respect to the origin. 
Corollary 4.3. Let $u$ be a solution of

$$
\left\{\begin{array}{l}
\Delta^{2} u=u^{p} \\
u>0
\end{array}\right.
$$

where $1<p<\frac{n+4}{n-4}$. Then $u(x) \leq c|x|^{-\frac{4}{p-1}}$ for $|x| \leq \frac{1}{2}$, where $c$ is a constant, depending on $n$ a nd $p$ only.

Corollary 4.3 is an immediate consequence of Theorem 1.3 and a blow-up argument due to R. Schoen for the equation (1.3) (for example, please see [P].) We omit the details of the proof.

\section{References}

[BCY] T. Branson, S-Y. A. Chang and P. C. Yang, Estimates and extremal problems for the log-determinant on 4-manifolds, Communication Math. Physics 149 (2) (1992), 241-262.

[BM] H. Brezis and F. Merle, Uniform estimates and blow-up behavior for solutions of $-\Delta u=$ $V(x) e^{u}$ in two dimensions, Comm. Partial Differential Equations 16 (1991), 1223-1253.

[BO] T. Branson and B. Orsted, Explicit functional determinants in four dimensions, Proc. Amer. Math. Soc. 113 (1991), 669-682.

[CGS] L. Caffarelli, B. Gidas and J. Spruck, Asymptotic Symmetry and local behavior of semilinear elliptic equations with critical Sobolev growth, Comm. Pure Appl. Math. 42 (1989), 271297.

[CL] Chen, E. and Li, C., Classification of solutions of some nonlinear elliptic equations, Duke Math. J. 63 (3) (1991), 615-622.

[CLn] C.C. Chen and C.S. Lin, Local behavior of singular positive solutions of semilinear elliptic equations with Sobolev exponent, Duke Math. J. 78 (1995), 315-334.

[CY] S-Y. A. Chang and P. C. Yang, Extremal metrics of zeta functional determinants on 4manifolds, Annals of Math. 142 (1995), 1171-212.

[GNN] B. Gidas, W.M. Ni, and L. Nirenberg, Symmetry of positive solutions of nonlinear equations in $\mathbf{R}^{n}$. Analysis and Applications part A, pp. 369-402, Advances in Math. Supp. Stud. 79, Academic Press, New York-London 1981.

[GT] D. Gilbarg, N. S, Trudinger, Elliptic partial differential equations, 2nd edition, Grundlehren der mathematischen Wissenschaften 224, Springer-Verlag, 1983.

[L] P. L. Lions, The concentration-compactness principle in the calculus of variations. The locally compact case, parts 1 and 2. Ann. Inst. H. Poincaré Anal. Non Linéaire. 1 (1984), no. 2, 109-145, no. 4, 223-283.

[P] D. Pollack, Compactness results for complete metrics of constant positive scalar curvature on subdomains of $S^{n}$, Indiana Univ. Math. J. 42 (1993), 1441-1456.

Chang-Shou Lin

Department of Mathematics

National Chung Cheng University

Ming-Hsiung, Chia-Yi, Taiwan

e-mail: cslin@math.ccu.edu.tw

(Received: September 26, 1996 ) 